\title{
Approximation of common solutions for variational inequalities and fixed point of strict pseudo-contractions in $q$-uniformly smooth Banach spaces
}

\section{Esmaeil Nazari", Saeid Askari and Mehdi Ramezani}

"Correspondence:

nazari.esmaeil@gmail.com

Department of Mathematics,

Tafresh University, Tafresh, Iran

\begin{abstract}
In the present paper, we introduce a general iterative algorithm for finding a common element of the set of common fixed points of an infinite family of strict pseudo-contractions and the set of solutions of the variational inequalities for finite family of strongly accretive mappings in a q-uniformly smooth Banach space. Furthermore, we prove strong convergence of the iterative sequence under suitable conditions. Our results generalize some recent results.

MSC: 47H09; 47H10;49J05
\end{abstract}

Keywords: fixed point; q-uniformly smooth Banach space; variational inequality; iterative algorithm; inverse strongly accretive operator

\section{Introduction}

Throughout this paper, we always assume that $X$ is a real Banach space with the dual $X^{*}$. Let $C$ be a subset of $X$, and $T$ be a self-mapping of $C$. We use $F(T)$ to denote the fixed points of $T$. For $q>1$, the generalized duality mapping $J_{q}: X \rightarrow 2^{X^{*}}$ is defined by

$$
J_{q}(x)=\left\{f \in X^{*}:\langle x, f\rangle=\|x\|^{q},\|f\|=\|x\|^{q-1}\right\},
$$

where $\langle\cdot, \cdot\rangle$ denotes the duality pairing between $X$ and $X^{*}$. In particular, $J_{q}=J_{2}$ is called the normalized duality mapping and $J_{q}(x)=\|x\|^{q-2} J_{2}(x)$ for $x \neq 0$. If $X:=H$ is a real Hilbert space, then $J=I$ where $I$ is the identity mapping. It is well known that if $X$ is smooth, then $J_{q}$ is single-valued, which is denoted by $j_{q}[1]$.

Let $U=\{x \in X:\|x\|=1\}$. A Banach space $X$ is said to be strictly convex if $\frac{\|x+y\|}{2} \leq 1$ for all $x, y \in X$ with $\|x\|=\|y\|=1$ and $x \neq y$. It is also called uniformly convex if lim $\| x_{n}-$ $y_{n} \|=0$ for any two sequences $\left\{x_{n}\right\},\left\{y_{n}\right\}$ in $X$ such that $\left\|x_{n}\right\|=\left\|y_{n}\right\|=1$ and $\lim \left\|\frac{x_{n}+y_{n}}{2}\right\|=1$. A Banach space $X$ is said to be Gâteaux differentiable if the limit

$$
\lim _{t \rightarrow 0} \frac{\|x+t y\|-\|x\|}{t}
$$

\section{Springer}

(0) 2015 Nazari et al.: licensee Springer. This is an Open Access article distributed under the terms of the Creative Commons Attribution License (http://creativecommons.org/licenses/by/4.0), which permits unrestricted use, distribution, and reproduction in any medium, provided the original work is properly credited. 
exists for all $x, y \in U$. In this case $X$ is smooth. Also, we define a function $\rho_{X}:[0, \infty) \rightarrow$ $[0, \infty)$ called the modulus of smoothness of $X$ as follows:

$$
\rho_{X}(t)=\sup \left\{\frac{1}{2}(\|x+y\|+\|x-y\|)-1: x \in U,\|y\|<t\right\} .
$$

A Banach space $X$ is said to be uniformly smooth if $\frac{\rho_{X}(t)}{t} \rightarrow 0$ as $t \rightarrow 0$. Suppose that $q>1$, then $X$ is said to be $q$-uniformly smooth if there exists $c>0$ such that $\rho_{X}(t) \leq c t^{q}$. It is easy to see that if $X$ is $q$-uniformly smooth, then $q \leq 2$ and $X$ is uniformly smooth.

Let $C$ be a nonempty, closed, and convex subset of a Banach space $X$ and $D$ be a nonempty subset of $C$, then a mapping $Q: C \rightarrow D$ is said to be sunny provided

$$
Q(Q x+t(x-Q x))=Q x
$$

whenever $Q x+t(x-Q x) \in C$ for $x \in C$, and $t \geq 0$. A mapping $Q: C \rightarrow D$ is called a retraction if $Q x=x$ for all $x \in D$. Furthermore, $Q$ is a sunny nonexpansive retraction from $C$ onto $D$ if $\mathrm{Q}$ is a retraction from $\mathrm{C}$ onto $\mathrm{D}$ which is also sunny and nonexpansive.

A subset $D$ of $C$ is called a sunny nonexpansive retraction of $C$ if there exists a sunny nonexpansive retraction from $C$ onto $D$. In real Hilbert space, a sunny nonexpansive retraction $Q_{C}$ coincides with the metric projection from $X$ onto $C$.

Definition 1.1 A mapping $T: C \rightarrow C$ is said to be:

(i) $\lambda$-strictly pseudo contractive [2], if for all $x, y \in C$ there exist $\lambda>0$ and $j_{q}(x-y) \in J_{q}(x-y)$ such that

$$
\left\langle T x-T y, j_{q}(x-y)\right\rangle \leq\|x-y\|^{q}-\lambda\|(I-T) x-(I-T) y\|^{q},
$$

or equivalently

$$
\left\langle(I-T) x-(I-T) y, j_{q}(x-y)\right\rangle \geq \lambda\|(I-T) x-(I-T) y\|^{q} .
$$

(ii) $L$-Lipschitzian if for all $x, y \in C$, there exists a constant $L>0$ such that

$$
\|T x-T y\| \leq L\|x-y\| .
$$

If $0<L<1$, then $T$ is a contraction, and if $L=1$, then $T$ is a nonexpansive mapping.

Remark 1.2 Let $C$ be a nonempty subset of a real Hilbert space $H$ and $T: C \rightarrow C$ be a mapping. Then $T$ is said to be $k$-strictly pseudocontractive [2], if for all $x, y \in C$, there exists constant $k \in[0,1)$ such that

$$
\|T x-T y\|^{2} \leq\|x-y\|+k\|(I-T) x-(I-T) y\|^{2}
$$

Definition 1.3 A mapping $F: C \rightarrow X$ is said to be accretive if for all $x, y \in C$ there exists $j_{q}(x-y) \in J_{q}(x-y)$ such that

$$
\left\langle F x-F y, j_{q}(x-y)\right| \geq 0 .
$$


For some $\eta>0, F: C \rightarrow X$ is said to be $\eta$-strongly accretive if for all $x, y \in C$ there exists $j_{q}(x-y) \in J_{q}(x-y)$ such that

$$
\left\langle F x-F y, j_{q}(x-y)\right\rangle \geq \eta\|x-y\|^{q} .
$$

For some $\mu>0$, the mapping $F: C \rightarrow X$ is said to be $\mu$-inverse strongly accretive if for all $x, y \in C$ there exists $j_{q}(x-y) \in J_{q}(x-y)$ such that

$$
\left\langle F x-F y, j_{q}(x-y)\right\rangle \geq \mu\|F x-F y\|^{q} .
$$

Note that if $X:=H$ is a real Hilbert space, accretive and strongly accretive operators coincide with monotone and strongly monotone operators, respectively.

Let $C$ be a nonempty, closed, and convex subset of $X$, and $A: C \rightarrow X$ be a mapping. The classical variational inequality problem is to find $x^{*} \in C$ such that

$$
\left\langle A x^{*}, j_{q}\left(x-x^{*}\right)\right| \geq 0, \quad \forall x \in C,
$$

where $j_{q}\left(x-x^{*}\right) \in J_{q}\left(x-x^{*}\right)$. The solution set of a variational inequality is denoted by $V I(C, A)$. If $X=: H$ is a real Hilbert space, the variational inequality problem reduces to find $x^{*} \in C$ such that

$$
\left\langle A x^{*}, x-x^{*}\right\rangle \geq 0, \quad \forall x \in C
$$

For more details of the variational inequality and its applications, we recommend the reader $[3,4]$. On the other hand, we note that the iterative approximations of fixed points for nonexpansive mappings have been extensively studied by many authors [5-9].

In order to find the common element of the solution set of a variational inclusion (3) and the set of fixed points of a nonexpansive mapping, Takahashi and Toyoda [10] introduced the following iterative scheme in a Hilbert space $H$. Starting with an arbitrary point $x_{1}=$ $x \in H$, define sequences $\left\{x_{n}\right\}$ by

$$
x_{n+1}=\alpha_{n} x_{n}+\left(1-\alpha_{n}\right) S P_{C}\left(x_{n}-\lambda_{n} A x_{n}\right),
$$

where $A: H \rightarrow H$ is an $\alpha$-inverse-strongly monotone mapping, $S: C \rightarrow C$ is a nonexpansive mapping and $\left\{\alpha_{n}\right\}$ is a sequence in $[0,1]$. Under mild conditions, they obtained a weak convergence theorem.

On the other hand, Aoyama et al. [11] considered the following algorithm in a uniformly convex and 2-uniformly smooth Banach spaces. For $x_{1}=x \in C$,

$$
x_{n+1}=\alpha_{n} x_{n}+\left(1-\alpha_{n}\right) Q_{C}\left(x_{n}-\lambda_{n} A x_{n}\right),
$$

where $Q_{C}: X \rightarrow C$ is a sunny nonexpansive retraction, and $A$ is a $\beta$-Lipschitzian and $\eta$-inverse strongly accretive operator. They proved that $\left\{x_{n}\right\}$ generated by (5) converges weakly to a unique element $z$ of $V I(C, A)$. 
Let $C$ be a nonempty, closed, and convex subset of a real $q$-uniformly smooth uniformly convex Banach space $X$. Assume the mapping $A_{m}: C \rightarrow X$ be a $\mu_{m}$-inverse-strongly accretive mapping for each $1 \leq m \leq r$, where $r$ is a positive integer. Let $\left\{T_{n}\right\}_{n=1}^{\infty}: C \rightarrow C$ be a family of $\lambda$-strict pseudo-contractions with $0<\lambda<1$. Define a mapping $S_{n} x:=\left(1-\gamma_{n}\right) x+\gamma_{n} T_{n} x$ for all $x \in C$ and $n \geq 1$.

In this paper, motivated by the works mentioned above, we consider the following iteration:

$$
\left\{\begin{array}{l}
x_{1} \in C \\
y_{n}=\alpha_{n} x_{n}+\left(1-\alpha_{n}\right) \sum_{m=1}^{r} \eta_{n}^{m} Q_{C}\left(x_{n}-\lambda_{m} A_{m} x_{n}\right) \\
x_{n+1}=Q_{C}\left[\beta_{n} \gamma f x_{n}+\left(I-\beta_{n} \mu F\right) S_{n} y_{n}\right], \quad n \geq 1
\end{array}\right.
$$

and we prove that the proposed iterative algorithm is strongly convergent under some mild conditions imposed on the algorithm parameters. The results proved in this paper represent a refinement and improvement of the previously found results in the earlier and recent literature.

\section{Preliminaries}

In order to prove our main results, we need the following lemmas.

Lemma 2.1 [12,13] Let $C$ be a closed convex subset of a smooth Banach space X. Let D be a nonempty subset of $C$. Let $Q: C \rightarrow D$ be a retraction and $J$ be the normalized duality mapping on $X$. Then the following are equivalent:

(a) $Q$ is sunny and nonexpansive.

(b) $\|Q x-Q y\|^{2} \leq\langle x-y, J(Q x-Q y)\rangle, \forall x, y \in C$.

(c) $\langle x-Q x, J(y-Q x)\rangle \leq 0, \forall x \in C, y \in D$.

(d) $\left\langle x-Q x, J_{q}(y-Q x)\right\rangle \leq 0, \forall x \in C, y \in D$.

Lemma 2.2 [14] Let $C$ be a closed convex subset of a strictly convex Banach space X. Let $T_{1}$ and $T_{2}$ be two nonexpansive mappings from $C$ into itself with $F\left(T_{1}\right) \cap F\left(T_{2}\right) \neq \emptyset$. Define a mapping $S$ by

$$
S x=k T_{1} x+(1-k) T_{2} x, \quad \forall x \in C,
$$

where $k$ is a constant in $(0,1)$. Then $S$ is nonexpansive and $F(S)=F\left(T_{1}\right) \cap F\left(T_{2}\right)$.

Lemma 2.3 [15] Let $\left\{s_{n}\right\}$ be a sequence of nonnegative real numbers satisfying

$$
s_{n+1}=\left(1-a_{n}\right) s_{n}+a_{n} b_{n}+c_{n},
$$

where $\left\{a_{n}\right\},\left\{b_{n}\right\},\left\{c_{n}\right\}$ satisfy the restrictions:

(i) $\lim _{n \rightarrow \infty} a_{n}=0, \sum_{n=1}^{\infty} a_{n}=\infty$,

(ii) $c_{n} \geq 0, \sum_{n=1}^{\infty} c_{n}<\infty$,

(iii) $\lim \sup _{n \rightarrow \infty} b_{n} \leq 0$.

Then $\lim _{n \rightarrow \infty} s_{n}=0$. 
Lemma 2.4 [16] Suppose that $q>1$. Then the following inequality holds:

$$
a b \leq \frac{1}{q} a^{q}+\left(\frac{q-1}{q}\right) b^{\frac{q}{q-1}}
$$

for arbitrary positive real numbers $a, b$.

Lemma 2.5 [17] Let $X$ be a real q-uniformly smooth Banach space, then there exists a constant $C_{q}>0$ such that

$$
\|x+y\|^{q} \leq\|x\|^{q}+q\left\langle y, J_{q}(x)\right\rangle+c_{q}\|y\|^{q}
$$

for all $x, y \in X$. In particular, if $X$ is real2-uniformly smooth Banach space, then there exists a best smooth constant $K>0$ such that

$$
\|x+y\|^{2} \leq\|x\|^{2}+2\langle y, J(x)\rangle+2 K\|y\|^{2}
$$

for all $x, y \in C$.

Lemma 2.6 [18] Let $X$ a real smooth and uniformly convex Banach space and let $r>0$. Then there exists a strictly increasing, continuous, and convex function $g:[0,2 r] \rightarrow R$ such that $g(0)=0$ and $g(\|x-y\|) \leq\|x\|^{2}-2\langle x, J y\rangle+\|y\|^{2}$, for all $x, y \in B_{r}$, where $B_{r}=\{z \in X$ : $\|z\| \leq r\}$.

Definition 2.7 [11] Let $T_{n}$ be a family of mappings from a subset $C$ of a Banach space $X$ into itself with $\bigcap_{n=1}^{\infty} F\left(T_{n}\right) \neq \emptyset$. We say that $\left\{T_{n}\right\}$ satisfies the AKTT-condition if for each bounded subset $B$ of $C$,

$$
\sum_{n=1}^{\infty} \sup _{\omega \in B}\left\|T_{n+1} \omega-T_{n} \omega\right\|<\infty .
$$

Lemma 2.8 [11] Suppose that $\left\{T_{n}\right\}$ satisfies the AKTT-condition such that:

(i) For each $x \in C,\left\{T_{n} x\right\}$ is converge strongly to some point in $C$.

(ii) Let the mapping $T: C \rightarrow C$ defined by $T x=\lim _{n \rightarrow \infty} T_{n} x$, for all $x \in C$.

Then $\lim _{n \rightarrow \infty} \sup _{\omega \in B}\left\|T \omega-T_{n} \omega\right\|=0$, for each bounded subset $B$ of $C$.

Lemma 2.9 $[7,8]$ Let $C$ be a closed and convex subset of a smooth Banach space X. Suppose that $\left\{T_{n}\right\}_{n=1}^{\infty}: C \rightarrow X$ is a family of $\lambda$-strictly pseudocontractive mappings; $\left\{\mu_{m}\right\}_{m=1}^{\infty}$ is a real sequence in $(0,1)$ such that $\sum_{n=1}^{\infty} \mu_{m}=1$. Then the following conclusions hold:

(i) A mapping $G: C \rightarrow X$ defined by $G:=\sum_{n=1}^{\infty} \mu_{n} T_{n}$ is a $\lambda$-strictly pseudocontractive mapping.

(ii) $F(G)=\bigcap_{n=1}^{\infty} F\left(T_{n}\right)$.

Lemma 2.10 [19] Let $C$ be a nonempty, closed, and convex subset of a real q-uniformly smooth Banach space $X$ which admits weakly sequentially continuous generalized duality mapping $j_{q}$ from $X$ into $X^{*}$. Let $T: C \rightarrow C$ be a nonexpansive mapping. Then, for all $\left\{x_{n}\right\} \subset C$, if $x_{n} \rightarrow x$ and $x_{n}-T x_{n} \rightarrow 0$, then $x=T x$. 
Lemma 2.11 [19] Let $C$ be a nonempty, closed, and convex subset of a real q-uniformly smooth Banach space X. Let $F: C \rightarrow E$ be a $k$-Lipschitzian and $\eta$-strongly accretive operator with constants $k, \eta>0$. Let $0<\mu<\left(\frac{q \eta}{C_{q} k^{q}}\right)^{\frac{1}{q-1}}$ and $\tau=\mu\left(\eta-\frac{C_{q} \mu^{q-1} k^{q}}{q}\right)$. Then for $t \in\left(0, \min \left\{1, \frac{1}{\tau}\right\}\right)$, the mapping $S: C \rightarrow E$ defined by $S:=(I-t \mu F)$ is a contraction with a constant $1-t \tau$.

Lemma 2.12 [19] Let $C$ be a nonempty, closed, and convex subset of a real q-uniformly smooth Banach space $X$. Let $Q_{C}$ be a sunny nonexpansive retraction from $X$ onto $C$. Let $F: C \rightarrow X$ be a $k$-Lipschitzian and $\eta$-strongly accretive operator with constants $k, \eta>0$, $f: C \rightarrow X$ be an L-Lipschitzian mapping with a constant $L \geq 0$ and $S: C \rightarrow C$ be a nonexpansive mapping such that $F(S) \neq \emptyset$. Let $0<\mu<\left(\frac{q \eta}{C_{q^{k q}}}\right)^{\frac{1}{q-1}}$ and $0 \leq \gamma L<\tau$, where $\tau=\mu\left(\eta-\frac{C_{q} \mu^{q-1} k^{q}}{q}\right)$. Then $\left\{x_{t}\right\}$ defined by

$$
x_{t}=Q_{C}\left[t \gamma f x_{t}+(I-t \mu F) S x_{t}\right]
$$

has the following properties:

(i) $\left\{x_{t}\right\}$ is bounded for each $t \in\left(0, \min \left\{1, \frac{1}{\tau}\right\}\right)$.

(ii) $\lim _{t \rightarrow 0}\left\|x_{t}-S x_{t}\right\|=0$.

(iii) $\left\{x_{t}\right\}$ defines a continuous curve from $\left(0, \min \left\{1, \frac{1}{\tau}\right\}\right)$ into $C$.

Lemma 2.13 [13] Let $C$ be a nonempty, closed, and convex subset of a real q-uniformly smooth Banach space $X$ which admits a weakly sequentially continuous generalized duality mapping $j_{q}$ from $X$ into $X^{*}$. Let $Q_{C}$ be a sunny nonexpansive retraction from $X$ onto $C$. Let $F: C \rightarrow X$ be a $k$-Lipschitzian and $\eta$-strongly accretive operator with constants $k, \eta>0$, $f: C \rightarrow X$ be an L-Lipschitzian mapping with a constant $L \geq 0$, and $S: C \rightarrow C$ be a nonexpansive mapping such that $F(S) \neq \emptyset$. Suppose that $0<\mu<\left(\frac{q \eta}{C_{q} k^{q}}\right)^{\frac{1}{q-1}}$ and $0 \leq \gamma L<\tau$, where $\tau=\mu\left(\eta-\frac{C_{q} \mu^{q-1} k^{q}}{q}\right)$. For each $t \in\left(0, \min \left\{1, \frac{1}{\tau}\right\}\right)$, let $\left\{x_{t}\right\}$ be defined by (8), then $\left\{x_{t}\right\}$ converges strongly to $x^{*} \in F(S)$ as $t \rightarrow 0$, in which $x^{*}$ is the unique solution of the variational inequality

$$
\left\langle(\mu F-\gamma V) x^{*}, j_{q}\left(x^{*}-p\right)\right\rangle \leq 0, \quad \forall p \in F(S)
$$

Lemma 2.14 [20] Let $X$ be a Banach space and J be a normality duality mapping. Then for any given $x, y \in X$, the following inequality holds:

$$
\|x+y\|^{2} \leq\|x\|^{2}+2\langle y, j(x+y)\rangle
$$

for all $j(x+y) \in J(x+y)$.

\section{Main results}

Theorem 3.1 Let $C$ be a nonempty, closed, and convex subset of a real q-uniformly smooth, uniformly convex Banach space $X$. Let $Q_{C}$ be a sunny nonexpansive retraction from $X$ onto $C$. Assume that the mapping $A_{m}: C \rightarrow H$ is a $\mu_{m}$-inverse-strongly accretive mapping for each $1 \leq m \leq r$, where $r$ is a positive integer. Let $F: C \rightarrow X$ be a $k$-Lipschitzian and $\eta$-strongly accretive operator with constants $k, \eta>0, f: C \rightarrow X$ be an L-Lipschitzian mapping with a constant $L \geq 0$. Suppose that $0<\mu<\left(\frac{q \eta}{C_{q} k^{q}}\right)^{\frac{1}{q-1}}$ and $0 \leq \gamma L<\tau$, where 
$\tau=\mu\left(\eta-\frac{C_{q} \mu^{q-1} k^{q}}{q}\right)$. Let $\left\{T_{n}\right\}_{n=1}^{\infty}: C \rightarrow C$ be a family of $\lambda$-strict pseudo-contractions with $0<\lambda<1$. Define a mapping $S_{n} x:=\left(1-\gamma_{n}\right) x+\gamma_{n} T_{n} x$, for all $x \in C$ and $n \geq 1$. Assume that $F:=\left(\bigcap_{m=1}^{r} V I\left(C, A_{m}\right)\right) \cap\left(\bigcap_{n=1}^{\infty} F\left(T_{n}\right)\right) \neq \emptyset$. Let $\left\{x_{n}\right\}$ be a sequence generated by the following iterative algorithm:

$$
\left\{\begin{array}{l}
x_{1} \in C \\
y_{n}=\alpha_{n} x_{n}+\left(1-\alpha_{n}\right) \sum_{m=1}^{r} \eta_{n}^{m} Q_{C}\left(x_{n}-\lambda_{m} A_{m} x_{n}\right), \\
x_{n+1}=Q_{C}\left[\beta_{n} \gamma f x_{n}+\left(I-\beta_{n} \mu F\right) S_{n} y_{n}\right], \quad n \geq 1
\end{array}\right.
$$

where $\left\{\alpha_{n}\right\},\left\{\beta_{n}\right\},\left\{\eta_{n}^{1}\right\},\left\{\eta_{n}^{2}\right\}, \ldots$ and $\left\{\eta_{n}^{r}\right\}$ are sequences in $(0,1)$ and $\lambda_{m}$ is a real number such that $0<\lambda_{m}<\left(\frac{q \mu_{m}}{C_{q}}\right)^{\frac{1}{q-1}}$, for each $1 \leq m \leq r$. Assume that the above control sequences satisfy the following restrictions:

(i) $\sum_{m=1}^{r} \eta_{n}^{m}=1, \forall n \geq 1, \sum_{n=1}^{\infty}\left|\eta_{n+1}^{m}-\eta_{n}^{m}\right|<\infty$.

(ii) $\lim _{n \rightarrow \infty} \eta_{n}^{m}=\eta^{m} \in(0,1)$, for each $m$, where $1 \leq m \leq r$.

(iii) $\sum_{n=1}^{\infty} \beta_{n}=\infty, \lim _{n \rightarrow \infty} \beta_{n}=0, \sum_{n=1}^{\infty}\left|\beta_{n+1}-\beta_{n}\right|<\infty$.

(iv) $\sum_{n=1}^{\infty}\left|\alpha_{n+1}-\alpha_{n}\right|<\infty, \liminf _{n \rightarrow \infty} \alpha_{n}>0$.

(v) $0 \leq \gamma_{n} \leq \delta, \delta=\min \left\{1,\left(\frac{q \lambda}{C_{q}}\right)^{\frac{1}{q-1}}\right\}$, and $\sum_{n=1}^{\infty}\left|\gamma_{n+1}-\gamma_{n}\right|<\infty$.

Suppose in addition that $\left\{T_{n}\right\}_{n=0}^{\infty}$ satisfies the AKTT-condition. Let $T: C \rightarrow C$ be the mapping defined by $T x=\lim _{n \rightarrow \infty} T_{n} x$ for all $x \in C$ and suppose that $F(T)=\bigcap_{n=0}^{\infty} F\left(T_{n}\right)$. Then the sequence $\left\{x_{n}\right\}$ converges strongly to $x^{*} \in F$ as $n \rightarrow \infty$, in which $x^{*}$ is the unique solution of the variational inequality,

$$
\left\langle(\mu F-\gamma f) x^{*}, j_{q}\left(x^{*}-p\right)\right\rangle \leq 0, \quad \forall p \in F(S)
$$

Proof We divide the proof into several steps.

Step 1 . We show that $I-\lambda_{m} A_{m}$ is nonexpansive for each $m$. Indeed, from Lemma 2.4, for all $x, y \in C$ we have

$$
\begin{aligned}
\|(I & \left.-\lambda_{m} A_{m}\right) x-\left(I-\lambda_{m} A_{m}\right) y \|^{q} \\
& =\left\|(x-y)-\lambda_{m}\left(A_{m} x-A_{m} y\right)\right\|^{q} \\
& \leq\|x-y\|^{q}-q \lambda_{m}\left\langle A_{m} x-A_{m} y, j_{q}(x-y)\right\rangle+C_{q} \lambda_{m}^{q}\left\|A_{m} x-A_{m} y\right\|^{q} \\
& \leq\|x-y\|^{q}-q \mu_{m} \lambda_{m}\left\|A_{m} x-A_{m} y\right\|^{q}+C_{q} \lambda_{m}^{q}\left\|A_{m} x-A_{m} y\right\|^{q} \\
& \leq\|x-y\|^{q}-\lambda_{m}\left(q \mu_{m}-C_{q} \lambda_{m}^{q-1}\right)\left\|A_{m} x-A_{m} y\right\|^{q} .
\end{aligned}
$$

It is clear that if $0<\lambda_{m} \leq\left(\frac{q \mu_{m}}{C_{q}}\right)^{\frac{1}{q-1}}$, then $I-\lambda_{m} A_{m}$ is nonexpansive for each $1 \leq m \leq r$.

Now, for each $1 \leq m \leq r$, put

$$
k_{n}^{m}=Q_{C}\left(x_{n}-\lambda_{m} A_{m} x_{n}\right), \quad z_{n}=\sum_{m=1}^{r} \eta_{n}^{m} k_{n}^{m} .
$$

Let $x^{*} \in F$, we have

$$
\begin{aligned}
\left\|k_{n}^{m}-x^{*}\right\| & =\left\|Q_{C}\left(x_{n}-\lambda_{m} A_{m} x_{n}\right)-Q_{C}\left(x^{*}-\lambda_{n} A_{m} x^{*}\right)\right\| \\
& \leq\left\|x_{n}-x^{*}\right\| \quad \forall m, 1 \leq m \leq r .
\end{aligned}
$$


On the other hand we have

$$
\begin{aligned}
\left\|y_{n}-x^{*}\right\| & =\left\|\alpha_{n} x_{n}+\left(1-\alpha_{n}\right) \sum_{m=1}^{r} \eta_{n}^{m} k_{n}^{m}-x^{*}\right\| \\
& \leq \alpha_{n}\left\|x_{n}-x^{*}\right\|+\left(1-\alpha_{n}\right) \sum_{m=1}^{r} \eta_{n}^{m}\left\|x_{n}-x^{*}\right\| \\
& =\alpha_{n}\left\|x_{n}-x^{*}\right\|+\left(1-\alpha_{n}\right)\left\|x_{n}-x^{*}\right\|=\left\|x_{n}-x^{*}\right\| .
\end{aligned}
$$

From (10) and the fact that $S_{n}$ is nonexpansive [19] we have

$$
\begin{aligned}
\left\|x_{n+1}-x^{*}\right\| & =\left\|Q_{C}\left(\beta_{n} \gamma f x_{n}+\left(I-\beta_{n} \mu F\right) S_{n} y_{n}\right)-Q_{C} x^{*}\right\| \\
& \leq\left\|\beta_{n} \gamma f x_{n}+\left(I-\beta_{n} \mu F\right) S_{n} y_{n}-x^{*}\right\| \\
& =\left\|\beta_{n}\left(\gamma f x_{n}-\mu F x^{*}\right)+\left(I-\beta_{n} \mu F\right)\left(S_{n} y_{n}-x^{*}\right)\right\| \\
& \leq \beta_{n}\left\|\gamma f x_{n}-\mu F x^{*}\right\|+\left(1-\beta_{n} \tau\right)\left\|S_{n} y_{n}-x^{*}\right\| \\
& \leq \beta_{n} \gamma\left\|f x_{n}-f x^{*}\right\|+\beta_{n}\left\|\gamma f x^{*}-\mu F x^{*}\right\|+\left(1-\beta_{n} \tau\right)\left\|y_{n}-x^{*}\right\| \\
& \leq \beta_{n} L \gamma\left\|x_{n}-x^{*}\right\|+\beta_{n}\left\|\gamma f x^{*}-\mu F x^{*}\right\|+\left(1-\beta_{n} \tau\right)\left\|x_{n}-x^{*}\right\| \\
& \leq\left(1-\beta_{n}(\tau-L \gamma)\right)\left\|x_{n}-x^{*}\right\|+\beta_{n}\left\|\gamma f x^{*}-\mu F x^{*}\right\| \\
& \leq \max \left\{\left\|x_{n}-x^{*}\right\|,(\tau-\gamma L)^{-1}\left\|\gamma f x^{*}-\mu F x^{*}\right\|\right\} .
\end{aligned}
$$

By induction, we find that

$$
\left\|x_{n+1}-x^{*}\right\| \leq \max \left\{\left\|x_{0}-x^{*}\right\|,(\tau-\gamma L)^{-1}\left\|\gamma f x^{*}-\mu F x^{*}\right\|\right\} .
$$

This shows that $\left\{x_{n}\right\}$ is bounded. Hence by (10), $\left\{y_{n}\right\}$ is also bounded.

Step 2: We show that $\lim _{n \rightarrow \infty}\left\|x_{n+1}-x_{n}\right\|=0$. Since

$$
\left\|k_{n+1}^{m}-k_{n}^{m}\right\|=\left\|Q_{C}\left(I-\lambda_{m} A_{m}\right) x_{n+1}-Q_{C}\left(I-\lambda_{m} A_{m}\right) x_{n}\right\| \leq\left\|x_{n+1}-x_{n}\right\| \quad \forall 1 \leq m \leq r .
$$

On the other hand, we have

$$
\begin{aligned}
\left\|z_{n+1}-z_{n}\right\| & =\left\|\sum_{m=1}^{r} \eta_{n+1}^{m} k_{n+1}^{m}-\sum_{m=1}^{r} \eta_{n}^{m} k_{n}^{m}\right\| \\
& \leq\left\|\sum_{m=1}^{r} \eta_{n+1}^{m} k_{n+1}^{m}-\sum_{m=1}^{r} \eta_{n+1}^{m} k_{n}^{m}\right\|+\left\|\sum_{m=1}^{r} \eta_{n+1}^{m} k_{n}^{m}-\sum_{m=1}^{r} \eta_{n}^{m} k_{n}^{m}\right\| \\
& \leq \sum_{m=1}^{r} \eta_{n+1}^{m}\left\|k_{n+1}^{m}-k_{n}^{m}\right\|+\sum_{m=1}^{r}\left|\eta_{n+1}^{m}-\eta_{n}^{m}\right|\left\|k_{n}^{m}\right\| \\
& \leq\left\|x_{n+1}-x_{n}\right\|+M \sum_{m=1}^{r}\left|\eta_{n+1}^{m}-\eta_{n}^{m}\right|,
\end{aligned}
$$

where $M$ is an appropriate constant such that

$$
M=\max \left\{\sup \left\{\left\|P_{C}\left(I-\lambda_{m} A_{m}\right) x_{n}\right\|: n \geq 1\right\}: 1 \leq m \leq r\right\} .
$$


Observe that

$$
y_{n+1}-y_{n}=\left(\alpha_{n+1}-\alpha_{n}\right)\left(x_{n+1}-z_{n}\right)+\alpha_{n}\left(x_{n+1}-x_{n}\right)+\left(1-\alpha_{n+1}\right)\left(z_{n+1}-z_{n}\right) .
$$

It follows from (11) that

$$
\begin{aligned}
\left\|y_{n+1}-y_{n}\right\| \leq & \left|\alpha_{n+1}-\alpha_{n}\right|\left\|x_{n+1}-z_{n}\right\|+\alpha_{n+1}\left\|x_{n+1}-x_{n}\right\|+\left(1-\alpha_{n+1}\right)\left\|z_{n+1}-z_{n}\right\| \\
\leq & \left|\alpha_{n+1}-\alpha_{n}\right|\left\|x_{n+1}-z_{n}\right\|+\alpha_{n+1}\left\|x_{n+1}-x_{n}\right\| \\
& \quad+\left(1-\alpha_{n+1}\right)\left(\left\|x_{n+1}-x_{n}\right\|+M \sum_{m=1}^{r}\left|\eta_{n+1}^{m}-\eta_{n}^{m}\right|\right) \\
\leq & \left|\alpha_{n+1}-\alpha_{n}\right|\left\|x_{n+1}-z_{n}\right\|+\left\|x_{n+1}-x_{n}\right\|+M \sum_{m=1}^{r}\left|\eta_{n+1}^{m}-\eta_{n}^{m}\right| .
\end{aligned}
$$

Note that

$$
\begin{aligned}
\left\|S_{n+1} y_{n+1}-S_{n} y_{n}\right\| \leq & \left\|S_{n+1} y_{n+1}-S_{n+1} y_{n}\right\|+\left\|S_{n+1} y_{n}-S_{n} y_{n}\right\| \\
& \leq\left\|y_{n+1}-y_{n}\right\|+\left\|\left(1-\gamma_{n+1}\right) y_{n}+\gamma_{n+1} T_{n} y_{n}-\left[\left(1-\gamma_{n}\right) y_{n}+\gamma_{n} T_{n} y_{n}\right]\right\| \\
& \leq\left\|y_{n+1}-y_{n}\right\|+\left\|\left(\gamma_{n+1}-\gamma_{n}\right)\left(T_{n+1} y_{n}-y_{n}\right)+\gamma_{n}\left(T_{n+1} y_{n}-T_{n} y_{n}\right)\right\| \\
& \leq\left\|y_{n+1}-y_{n}\right\|+\left|\gamma_{n+1}-\gamma_{n}\right|\left\|T_{n+1} y_{n}-y_{n}\right\|+\gamma_{n}\left\|T_{n+1} y_{n}-T_{n} y_{n}\right\| \\
& \leq\left|\alpha_{n+1}-\alpha_{n}\right|\left\|x_{n+1}-z_{n}\right\|+\left\|x_{n+1}-x_{n}\right\|+M \sum_{m=1}^{r}\left|\eta_{n+1}^{m}-\eta_{n}^{m}\right| \\
& \quad+\left|\gamma_{n+1}-\gamma_{n}\right|\left\|T_{n+1} y_{n}-y_{n}\right\|+\gamma_{n}\left\|T_{n+1} y_{n}-T_{n} y_{n}\right\| .
\end{aligned}
$$

On the other hand,

$$
\begin{aligned}
&\left\|x_{n+1}-x_{n}\right\| \\
&=\left\|Q_{C}\left(\beta_{n} \gamma f x_{n}+\left(I-\beta_{n} \mu F\right) S_{n} y_{n}\right)-Q_{C}\left(\beta_{n-1} \gamma f x_{n-1}+\left(I-\beta_{n-1} \mu F\right) S_{n-1} y_{n-1}\right)\right\| \\
& \leq\left\|\beta_{n} \gamma f x_{n}+\left(I-\beta_{n} \mu F\right) S_{n} y_{n}-\left(\beta_{n-1} \gamma f x_{n-1}+\left(I-\beta_{n-1} \mu F\right) S_{n-1} y_{n-1}\right)\right\| \\
& \leq \| \beta_{n} \gamma\left(f x_{n}-f x_{n-1}\right)+\left(\beta_{n}-\beta_{n-1}\right) \gamma f x_{n-1} \\
&+\left(I-\beta_{n} \mu F\right)\left(S_{n} y_{n}-S_{n-1} y_{n-1}\right)+\left(\beta_{n}-\beta_{n-1}\right) \mu F S_{n-1} y_{n-1} \| \\
& \leq \beta_{n} \gamma L\left\|x_{n}-x_{n-1}\right\|+\left|\beta_{n}-\beta_{n-1}\right|\left(\gamma\left\|f x_{n-1}\right\|+\mu\left\|F S_{n-1} y_{n-1}\right\|\right) \\
&+\left(1-\beta_{n} \tau\right)\left\|S_{n} y_{n}-S_{n-1} y_{n-1}\right\| .
\end{aligned}
$$

Substituting (13) into (14), we obtain

$$
\begin{aligned}
& \left\|x_{n+1}-x_{n}\right\| \\
& \leq \quad \beta_{n} \gamma L\left\|x_{n}-x_{n-1}\right\|+\left|\beta_{n}-\beta_{n-1}\right|\left(\gamma\left\|f x_{n-1}\right\|+\mu\left\|F S_{n-1} y_{n-1}\right\|\right) \\
& \quad+\left(1-\beta_{n} \tau\right)\left(\left|\alpha_{n}-\alpha_{n-1}\right|\left\|x_{n}-z_{n-1}\right\|+\left\|x_{n}-x_{n-1}\right\|+M \sum_{m=1}^{r}\left|\eta_{n}^{m}-\eta_{n-1}^{m}\right|\right.
\end{aligned}
$$




$$
\begin{aligned}
& \left.+\left|\gamma_{n}-\gamma_{n-1}\right|\left\|T_{n} y_{n-1}-y_{n-1}\right\|+\gamma_{n-1}\left\|T_{n} y_{n-1}-T_{n-1} y_{n-1}\right\|\right) \\
\leq & \left(1-\beta_{n}(\tau-\gamma L)\right)\left\|x_{n}-x_{n-1}\right\|+\left(\left|\beta_{n}-\beta_{n-1}\right|+\left|\alpha_{n}-\alpha_{n-1}\right|+\left|\gamma_{n}-\gamma_{n-1}\right|\right. \\
& \left.+\sum_{m=1}^{r}\left|\eta_{n}^{m}-\eta_{n-1}^{m}\right|\right) M_{1}+\left\|T_{n} y_{n-1}-T_{n-1} y_{n-1}\right\|
\end{aligned}
$$

where $M_{1}=\sup _{n \geq 0}\left\{\gamma\left\|f x_{n-1}\right\|+\mu\left\|F S_{n-1} y_{n-1}\right\|,\left\|x_{n}-z_{n-1}\right\|,\left\|T_{n} y_{n-1}-y_{n-1}\right\|, M\right\}$.

Since $\left\{T_{n}\right\}_{n=1}^{\infty}$ satisfies the AKTT-condition, we deduce that

$$
\sum_{n=0}^{\infty}\left\|T_{n} y_{n-1}-T_{n-1} y_{n-1}\right\| \leq \sum_{n=0}^{\infty} \sup _{\omega \in\left\{y_{n-1}\right\}}\left\|T_{n} \omega-T_{n-1} \omega\right\|<\infty .
$$

From (14), (16), and Lemma 2.3, we deduce that

$$
\lim _{n \rightarrow \infty}\left\|x_{n+1}-x_{n}\right\|=0 .
$$

We observe that

$$
\begin{aligned}
\left\|S_{n} y_{n}-x_{n}\right\| & \leq\left\|x_{n+1}-x_{n}\right\|+\left\|x_{n+1}-S_{n} y_{n}\right\| \\
& =\left\|x_{n+1}-x_{n}\right\|+\left\|Q_{C}\left(\beta_{n} \gamma f x_{n}+\left(I-\beta_{n} \mu F\right) S_{n} y_{n}\right)-S_{n} y_{n}\right\| \\
& =\left\|x_{n+1}-x_{n}\right\|+\left\|\left(\beta_{n} \gamma f x_{n}+\left(I-\beta_{n} \mu F\right) S_{n} y_{n}\right)-S_{n} y_{n}\right\| \\
& =\left\|x_{n+1}-x_{n}\right\|+\beta_{n}\left\|\gamma f x_{n}-\mu F S_{n} y_{n}\right\| .
\end{aligned}
$$

From the condition (iii) and (17), we have

$$
\lim _{n \rightarrow \infty}\left\|S_{n} y_{n}-x_{n}\right\|=0
$$

Step 3. We prove that $\lim _{n \rightarrow \infty}\left\|T_{n} x_{n}-x_{n}\right\|=0$.

From Lemma 2.5, we have

$$
\begin{aligned}
\left\|k_{n}^{m}-x^{*}\right\|^{q} & =\left\|Q_{C}\left(x_{n}-\lambda_{m} A_{m} x_{n}\right)-Q_{c}\left(x^{*}-\lambda_{m} A_{m} x^{*}\right)\right\|^{q} \\
& \leq\left\|\left(I-\lambda_{m} A_{m}\right) x_{n}-\left(I-\lambda_{m} A_{m}\right) x^{*}\right\|^{q} \\
& \leq\left\|x_{n}-x^{*}\right\|^{q}-\lambda_{m}\left(q \mu_{m}-C_{q} \lambda_{m}^{q-1}\right)\left\|A_{m} x_{n}-A_{m} x^{*}\right\|^{q}
\end{aligned}
$$

and

$$
\begin{aligned}
\left\|z_{n}-x^{*}\right\|^{q} & =\left\|\sum_{m=1}^{r} \eta_{n}^{m} k_{n}^{m}-x^{*}\right\|^{q} \leq \sum_{m=1}^{r} \eta_{n}^{m}\left\|k_{n}^{m}-x^{*}\right\|^{q} \\
& \leq \sum_{m=1}^{r} \eta_{n}^{m}\left(\left\|x_{n}-x^{*}\right\|^{q}-\lambda_{m}\left(q \mu_{m}-C_{q} \lambda_{m}^{q-1}\right)\left\|A_{m} x_{n}-A_{m} x^{*}\right\|^{q}\right) \\
& =\left\|x_{n}-x^{*}\right\|^{q}-\sum_{m=1}^{r} \eta_{n}^{m} \lambda_{m}\left(q \mu_{m}-C_{q} \lambda_{m}^{q-1}\right)\left\|A_{m} x_{n}-A_{m} x^{*}\right\|^{q} .
\end{aligned}
$$


By the convexity of $\|\cdot\|$, for all $q>1$, and Lemma 2.5 , we obtain

$$
\begin{aligned}
&\left\|x_{n+1}-x^{*}\right\|^{q} \\
&=\left\|Q_{C}\left(\beta_{n} \gamma f x_{n}+\left(I-\beta_{n} \mu F\right) S_{n} y_{n}\right)-x^{*}\right\|^{q} \\
& \leq\left\|\left(\beta_{n} \gamma f x_{n}+\left(I-\beta_{n} \mu F\right) S_{n} y_{n}\right)-x^{*}\right\|^{q} \\
&=\left\|\beta_{n}\left(\gamma f x_{n}-\mu F S_{n} y_{n}\right)+S_{n} y_{n}-x^{*}\right\|^{q} \\
& \leq\left\|S_{n} y_{n}-x^{*}\right\|^{q}+q\left(\beta_{n}\left(\gamma f x_{n}-\mu F S_{n} y_{n}\right), J_{q}\left(S_{n} y_{n}-x^{*}\right)\right\rangle+C_{q}\left\|\beta_{n}\left(\gamma f x_{n}-\mu F S_{n} y_{n}\right)\right\|^{q} \\
& \leq\left\|y_{n}-x^{*}\right\|^{q}+q \beta_{n}\left\|\gamma f x_{n}-\mu F S_{n} y_{n}\right\|\left\|S_{n} y_{n}-x^{*}\right\|^{q-1}+C_{q} \beta_{n}^{q}\left\|\gamma f x_{n}-\mu F S_{n} y_{n}\right\|^{q} \\
& \leq\left\|\beta_{n} x_{n}+\left(1-\beta_{n}\right) z_{n}-x^{*}\right\|^{q}+\beta_{n} M_{2} \\
& \leq\left\|\beta_{n}\left(x_{n}-x^{*}\right)+\left(1-\beta_{n}\right)\left(z_{n}-x^{*}\right)\right\|^{q}+\beta_{n} M_{2} \\
& \leq \beta_{n}\left\|x_{n}-x^{*}\right\|^{q}+\left(1-\beta_{n}\right)\left\|z_{n}-x^{*}\right\|^{q}+\beta_{n} M_{2}, \\
& \leq \beta_{n}\left\|x_{n}-x^{*}\right\|^{q}+\left(1-\beta_{n}\right)\left[\left\|x_{n}-x^{*}\right\|^{q}-\sum_{m=1}^{r} \eta_{n}^{m} \lambda_{m}\left(q \mu_{m}\right.\right. \\
& \leq\left.\left.-C_{q} \lambda_{m}^{q-1}\right)\left\|A_{m} x_{n}-A_{m} x^{*}\right\|^{q}\right]+\beta_{n} M_{2}, \\
& \\
& \\
& \\
& \\
&
\end{aligned}
$$

where

$$
M_{2}=\sup _{n \geq 0}\left\{q\left\|\gamma f x_{n}-\mu F S_{n} y_{n}\right\|\left\|S_{n} y_{n}-x^{*}\right\|^{q-1}+C_{q} \beta_{n}^{q-1}\left\|\gamma f x_{n}-\mu F S_{n} y_{n}\right\|^{q}\right\}<\infty
$$

By the fact that $a^{r}-b^{r} \leq r a^{r-1}(a-b), \forall r \geq 1$, we get

$$
\begin{aligned}
& \left(1-\beta_{n}\right) \sum_{m=1}^{r} \eta_{n}^{m} \lambda_{m}\left(q \mu_{m}-C_{q} \lambda_{m}^{q-1}\right)\left\|A_{m} x_{n}-A_{m} x^{*}\right\|^{q} \\
& \quad \leq\left\|x_{n}-x^{*}\right\|^{q}-\left\|x_{n+1}-x^{*}\right\|^{q}+\beta_{n} M_{2} \\
& \quad \leq q\left\|x_{n}-x^{*}\right\|^{q-1}\left(\left\|x_{n}-x^{*}\right\|-\left\|x_{n+1}-x^{*}\right\|\right)+\beta_{n} M_{2} \\
& \quad \leq q\left\|x_{n}-x^{*}\right\|^{q-1}\left\|x_{n}-x_{n+1}\right\|+\beta_{n} M_{2} .
\end{aligned}
$$

Since $0<\lambda_{m}<\left(\frac{q \mu_{m}}{C_{q}}\right)^{\frac{1}{q-1}}$, from (17) and (iii) and the fact that $\left\{x_{n}\right\}$ is bounded we have

$$
\lim _{n \rightarrow \infty}\left\|A_{m} x_{n}-A_{m} x^{*}\right\|=0, \quad \forall m, 1 \leq m \leq r
$$

Setting $r_{m}=\sup \left\{\left\|x_{n}-x^{*}\right\|,\left\|k_{n}^{m}-x^{*}\right\|\right\}$, we have from Lemmas 2.1 and 2.6

$$
\begin{aligned}
\left\|k_{n}^{m}-x^{*}\right\|^{2} & =\left\|Q_{C}\left(I-\lambda_{m} A_{m}\right) x_{n}-Q_{C}\left(I-\lambda_{m} A_{m}\right) x^{*}\right\|^{2} \\
& \leq\left\langle x_{n}-\lambda_{m} A_{m} x_{n}-\left(x^{*}-\lambda_{m} A_{m} x^{*}\right), j\left(k_{n}^{m}-x^{*}\right)\right\rangle
\end{aligned}
$$




$$
\begin{aligned}
\leq & \left\langle x_{n}-x^{*}, j\left(k_{n}^{m}-x^{*}\right)\right\rangle+\lambda_{m}\left\langle A_{m} x^{*}-A_{m} x_{n}, j\left(k_{n}^{m}-x^{*}\right)\right\rangle \\
\leq & \frac{1}{2}\left[\left\|x_{n}-x^{*}\right\|^{2}+\left\|k_{n}^{m}-x^{*}\right\|^{2}-g_{m}\left(\left\|x_{n}-x^{*}-k_{n}^{m}+x^{*}\right\|\right)\right] \\
& +\lambda_{m}\left\langle A_{m} x^{*}-A_{m} x_{n}, j\left(k_{n}^{m}-x^{*}\right)\right\rangle,
\end{aligned}
$$

where $g_{m}:\left[0,2 r_{m}\right) \rightarrow[0, \infty)$ is a continuous, strictly increasing, and convex function such that $g_{m}(0)=0$ for all $1 \leq m \leq r$. Hence, we have

$$
\left\|k_{n}^{m}-x^{*}\right\|^{2} \leq\left\|x_{n}-x^{*}\right\|^{2}-g_{m}\left(\left\|x_{n}-k_{n}^{m}\right\|\right)+2 \lambda_{m}\left\|A_{m} x^{*}-A_{m} x_{n}\right\|\left\|k_{n}^{m}-x^{*}\right\|
$$

for all $m$, with $1 \leq m \leq r$. On the other hand, we have

$$
\left\|z_{n}-x_{n}\right\|^{2} \leq\left\|\sum_{m=1}^{r} \eta_{n}^{m} k_{n}^{m}-x_{n}\right\|^{2} \leq \sum_{m=1}^{r} \eta_{n}^{m}\left\|k_{n}^{m}-x_{n}\right\|^{2} .
$$

Since $g_{m}$ is increasing and convex by using (20) we have

$$
\begin{aligned}
& g_{m}\left(\left\|z_{n}-x_{n}\right\|^{2}\right) \\
& \quad \leq g_{m}\left(\sum_{m=1}^{r} \eta_{n}^{m}\left\|k_{n}^{m}-x_{n}\right\|^{2}\right) \leq \sum_{m=1}^{r} \eta_{n}^{m} g_{m}\left(\left\|k_{n}^{m}-x_{n}\right\|^{2}\right) \\
& \quad \leq \sum_{m=1}^{r} \eta_{n}^{m}\left[\left\|x_{n}-x^{*}\right\|^{2}-\left\|k_{n}^{m}-x^{*}\right\|^{2}+2 \lambda_{m}\left\|A_{m} x^{*}-A_{m} x_{n}\right\|\left\|k_{n}^{m}-x^{*}\right\|\right] \\
& \quad=\left\|x_{n}-x^{*}\right\|^{2}-\sum_{m=1}^{r} \eta_{n}^{m}\left\|k_{n}^{m}-x^{*}\right\|^{2}+2 \sum_{m=1}^{r} \eta_{n}^{m} \lambda_{m}\left\|A_{m} x^{*}-A_{m} x_{n}\right\|\left\|k_{n}^{m}-x^{*}\right\| .
\end{aligned}
$$

Thus we have

$$
\sum_{m=1}^{r} \eta_{n}^{m}\left\|k_{n}^{m}-x^{*}\right\|^{2} \leq\left\|x_{n}-x^{*}\right\|^{2}-g_{m}\left(\left\|z_{n}-x_{n}\right\|^{2}\right)+2 \sum_{m=1}^{r} \eta_{n}^{m} \lambda_{m}\left\|A_{m} x^{*}-A_{m} x_{n}\right\|\left\|k_{n}^{m}-x^{*}\right\| .
$$

Thanks to Lemma 2.5 we have

$$
\begin{aligned}
& \left\|x_{n+1}-x^{*}\right\|^{2} \\
& \quad=\left\|Q_{C}\left(\beta_{n} \gamma f x_{n}+\left(I-\beta_{n} \mu F\right) S_{n} y_{n}\right)-x^{*}\right\|^{2} \\
& \quad \leq\left\|\left(\beta_{n} \gamma f x_{n}+\left(I-\beta_{n} \mu F\right) S_{n} y_{n}\right)-x^{*}\right\|^{2} \\
& \quad=\left\|\beta_{n}\left(\gamma f x_{n}-\mu F S_{n} y_{n}\right)+S_{n} y_{n}-x^{*}\right\|^{2} \\
& \quad \leq\left\|S_{n} y_{n}-x^{*}\right\|^{2}+2\left\langle\beta_{n}\left(\gamma f x_{n}-\mu F S_{n} y_{n}\right), j_{q}\left(\beta_{n}\left(\gamma f x_{n}-\mu F S_{n} y_{n}\right)+S_{n} y_{n}-x^{*}\right)\right\rangle \\
& \quad \leq\left\|y_{n}-x^{*}\right\|^{2}+\beta_{n} M_{3} \\
& \quad=\left\|\beta_{n} x_{n}+\left(1-\beta_{n}\right) z_{n}-x^{*}\right\|^{2}+\beta_{n} M_{3} \\
& \quad \leq \beta_{n}\left\|x_{n}-x^{*}\right\|^{2}+\left(1-\beta_{n}\right)\left\|z_{n}-x^{*}\right\|^{2}+\beta_{n} M_{3}
\end{aligned}
$$




$$
\begin{aligned}
\leq & \beta_{n}\left\|x_{n}-x^{*}\right\|^{2}+\left(1-\beta_{n}\right)\left(\left\|\sum_{m=1}^{r} \eta_{n}^{m} k_{n}^{m}-x^{*}\right\|\right)^{2}+\beta_{n} M_{3} \\
\leq & \beta_{n}\left\|x_{n}-x^{*}\right\|^{2}+\left(1-\beta_{n}\right) \sum_{m=1}^{r} \eta_{n}^{m}\left\|k_{n}^{m}-x^{*}\right\|^{2}+\beta_{n} M_{3} \\
\leq & \beta_{n}\left\|x_{n}-x^{*}\right\|^{2}+\left(1-\beta_{n}\right)\left(\left\|x_{n}-x^{*}\right\|^{2}-g_{m}\left(\left\|z_{n}-x_{n}\right\|^{2}\right)\right. \\
& \left.+2 \sum_{m=1}^{r} \eta_{n}^{m} \lambda_{m}\left\|A_{m} x^{*}-A_{m} x_{n}\right\|\left\|k_{n}^{m}-x^{*}\right\|\right)+\beta_{n} M_{3} \\
\leq & \left\|x_{n}-x^{*}\right\|^{2}-\left(1-\beta_{n}\right) g_{m}\left(\left\|z_{n}-x_{n}\right\|^{2}\right)+2\left(1-\beta_{n}\right) \sum_{m=1}^{r} \eta_{n}^{m} \lambda_{m} \\
& \times\left\|A_{m} x^{*}-A_{m} x_{n}\right\|\left\|k_{n}^{m}-x^{*}\right\|+\beta_{n} M_{3},
\end{aligned}
$$

where $M_{3}=\sup _{n \geq 0}\left\{2\left\langle\gamma f x_{n}-\mu F S_{n} y_{n}, j_{q}\left(\beta_{n}\left(\gamma f x_{n}-\mu f S_{n} y_{n}\right)+S_{n} y_{n}-x^{*}\right)\right\rangle\right\}$.

This in turn implies that

$$
\begin{aligned}
\left(1-\beta_{n}\right) g_{m}\left(\left\|z_{n}-x_{n}\right\|^{2}\right) \leq & \left\|x_{n}-x^{*}\right\|^{2}-\left\|x_{n+1}-x^{*}\right\|^{2} \\
& +2\left(1-\beta_{n}\right) \sum_{m=1}^{r} \eta_{n}^{m} \lambda_{m}\left\|A_{m} x^{*}-A_{m} x_{n}\right\|\left\|k_{n}^{m}-x^{*}\right\|+\beta_{n} M_{3} \\
\leq & \left\|x_{n}-x_{n+1}\right\|\left(\left\|x_{n}-x^{*}\right\|+\left\|x_{n+1}-x^{*}\right\|\right) \\
& +2\left(1-\beta_{n}\right) \sum_{m=1}^{r} \eta_{n}^{m} \lambda_{m}\left\|A_{m} x^{*}-A_{m} x_{n}\right\|\left\|k_{n}^{m}-x^{*}\right\|+\beta_{n} M_{3} .
\end{aligned}
$$

In view of (ii), (iii), (17), and (19) we have

$$
\lim _{n \rightarrow \infty} g_{m}\left(\left\|z_{n}-x_{n}\right\|^{2}\right)=0
$$

By the properties of $g_{m}$, we get

$$
\lim _{n \rightarrow \infty}\left\|z_{n}-x_{n}\right\|^{2}=0
$$

On the other hand,

$$
\begin{aligned}
\left\|S_{n} x_{n}-x_{n}\right\| & \leq\left\|S_{n} x_{n}-S_{n} y_{n}\right\|+\left\|S_{n} y_{n}-x_{n}\right\| \\
& \leq\left\|x_{n}-y_{n}\right\|+\left\|S_{n} y_{n}-x_{n}\right\| \\
& \leq\left\|x_{n}-z_{n}\right\|+\left\|z_{n}-y_{n}\right\|+\left\|S_{n} y_{n}-x_{n}\right\| \\
& =\left\|x_{n}-z_{n}\right\|+\beta_{n}\left\|x_{n}-z_{n}\right\|+\left\|S_{n} y_{n}-x_{n}\right\| .
\end{aligned}
$$

It follows from (21), (18), and (iii) that

$$
\lim _{n \rightarrow \infty}\left\|S_{n} x_{n}-x_{n}\right\|=0
$$


Next, we show that $\left\|x_{n}-S x_{n}\right\| \rightarrow 0$ as $n \rightarrow \infty$. For any bounded subset $B$ of $C$, we observe that

$$
\begin{aligned}
\sup \left\|S_{n+1} \omega-S_{n} \omega\right\|= & \sup _{\omega \in B}\left\|\gamma_{n+1} \omega+\left(1-\gamma_{n+1}\right) T_{n+1} \omega-\left(\gamma_{n} \omega+\left(1-\gamma_{n}\right) T_{n} \omega\right)\right\| \\
\leq & \left|\gamma_{n+1}-\gamma_{n}\right| \sup _{\omega \in B}|\omega|+\left(1-\gamma_{n+1}\right) \sup _{\omega \in B}\left\|T_{n+1} \omega-T_{n} \omega\right\| \\
& \quad+\left|\gamma_{n+1}-\gamma_{n}\right| \sup _{\omega \in B}\left\|T_{n} \omega\right\| \\
\leq & \left|\gamma_{n+1}-\gamma_{n}\right| M_{3}+\sup _{\omega \in B}\left\|T_{n+1} \omega-T_{n} \omega\right\|,
\end{aligned}
$$

where $M_{3}=\sup _{n \geq 1}\left\{\|\omega\|,\left\|T_{n} \omega\right\|\right\}$. By (v) and the fact that $\left\{T_{n}\right\}$ satisfies the AKTTcondition, we have

$$
\sum_{n=1}^{\infty} \sup _{\omega \in B}\left\|S_{n+1} \omega-S_{n} \omega\right\|<\infty,
$$

that is, $\left\{S_{n}\right\}$ satisfies the AKTT-condition. Now we define the nonexpansive mapping $S$ : $C \rightarrow C$ by $S x=\lim _{n \rightarrow \infty} S_{n} x$ for all $x \in C$. Since $\left\{\gamma_{n}\right\}$ is bounded, there exists a subsequence $\left\{\gamma_{n_{i}}\right\}$ of $\left\{\gamma_{n}\right\}$ such that $\gamma_{n_{i}} \rightarrow v$ as $i \rightarrow \infty$. It follows that

$$
S x=\lim _{i \rightarrow \infty} S_{n_{i}} x=\lim _{i \rightarrow \infty}\left[\gamma_{n_{i}} x+\left(1-\gamma_{n_{i}}\right) T_{n_{i}} x\right]=v x+(1-v) T x, \quad \forall x \in C .
$$

That is $F(S)=F(T)$. Hence $F(S)=\bigcap_{n=1}^{\infty} F\left(T_{n}\right)=\bigcap_{n=1}^{\infty} F\left(S_{n}\right)$. On the other hand we have

$$
\begin{aligned}
\left\|x_{n}-S x_{n}\right\| & \leq\left\|x_{n}-S_{n} x_{n}\right\|+\left\|S_{n} x_{n}-S x_{n}\right\| \\
& \leq\left\|x_{n}-S_{n} x_{n}\right\|+\sup _{\omega \in\left\{x_{n}\right\}}\left\|S_{n} \omega-S \omega\right\| .
\end{aligned}
$$

This implies by Lemma 2.8 and (22) that

$$
\lim _{n \rightarrow \infty}\left\|x_{n}-S x_{n}\right\|=0
$$

Now we define a mapping $h: C \rightarrow C$ by

$$
h x=\sum_{m=1}^{r} \eta^{m} P_{C}\left(I-\lambda_{m} A_{m}\right) x, \quad \forall x \in C,
$$

where $\eta^{m}=\lim _{n \rightarrow \infty} \eta_{n}^{m}$. From Lemma 2.9, $h$ is nonexpansive such that

$$
F(h)=\bigcap_{m=1}^{r} F\left(P_{C}\left(I-\lambda_{m} A_{m}\right)\right)=\bigcap_{m=1}^{r} V I\left(C, A_{m}\right)=\Omega .
$$

Next, we define a mapping $U: C \rightarrow C$ by $U x=\delta S x+(1-\delta) h x$, where $\delta \in(0,1)$ is a constant. Then by Lemma $2.2, U$ is a nonexpansive and

$$
F(U)=F(S) \cap F(h)=\bigcap_{n=1}^{\infty} F\left(T_{n}\right) \cap \Omega=F=F(T) \cap \Omega .
$$


Note that

$$
\begin{aligned}
\left\|x_{n}-h x_{n}\right\| & \leq\left\|x_{n}-z_{n}\right\|+\left\|z_{n}-h x_{n}\right\| \\
& \leq\left\|x_{n}-z_{n}\right\|+\left\|\sum_{n=1}^{m} \eta_{n}^{m} P_{C}\left(I-\lambda_{m} A_{m}\right) x_{n}-\sum_{m=1}^{r} \eta^{m} P_{C}\left(I-\lambda_{m} A_{m}\right) x_{n}\right\| \\
& \leq\left\|x_{n}-z_{n}\right\|+M \sum_{m=1}^{r}\left|\eta_{n}^{m}-\eta^{m}\right| .
\end{aligned}
$$

In view of restriction (ii), we find from (21) that

$$
\lim _{n \rightarrow \infty}\left\|x_{n}-h x_{n}\right\|=0
$$

Setting $x_{t}=Q_{C}\left[t \gamma f x_{t}+(I-t \mu F) U x_{t}\right]$, it follows from Lemma 2.13 that $\left\{x_{t}\right\}$ converges strongly to a point $x^{*} \in F(U)=F$, in which $x^{*}$ is the unique solution of the variational inequality (9). From (23) and (24), we have

$$
\begin{aligned}
\left\|x_{n}-U x_{n}\right\| & =\left\|\delta\left(x_{n}-S x_{n}\right)+(1-\delta)\left(x_{n}-h x_{n}\right)\right\| \\
& \leq \delta\left\|x_{n}-S x_{n}\right\|+(1-\delta)\left\|x_{n}-h x_{n}\right\| \rightarrow 0 .
\end{aligned}
$$

Step 4. We show that

$$
\lim \sup \left\{(\gamma f-\mu F) x^{*}, j_{q}\left(x_{n}-x^{*}\right)\right\rangle \leq 0,
$$

where $x^{*}$ is a solution of the variational inequality (9). To show this, we can choose a subsequence $\left\{x_{n_{j}}\right\}$ of $\left\{x_{n}\right\}$ such that

$$
\limsup _{n \rightarrow \infty}\left\langle(\gamma f-\mu F) x^{*}, j_{q}\left(x_{n}-x^{*}\right)\right\rangle=\lim _{j \rightarrow \infty}\left\langle(\gamma f-\mu F) x^{*}, j_{q}\left(x_{n_{j}}-x^{*}\right)\right\rangle .
$$

By reflexivity of a Banach space $X$ and since $\left\{x_{n}\right\}$ is bounded, there exists a subsequence $\left\{x_{n_{j}}\right\}$ of $\left\{x_{n}\right\}$ which converges weakly to $z$. Without loss of generality, we can assume that $x_{n_{j}} \rightarrow z$. Since $\left\|x_{n}-U x_{n}\right\| \rightarrow 0$ by step 3 , we obtain $z=U z$ and we have $z \in F(U)$. Since Banach space $X$ has a weakly sequentially continuous generalized duality mapping, we obtain

$$
\begin{aligned}
\limsup _{n \rightarrow \infty}\left\langle(\gamma f-\mu F) x^{*}, j_{q}\left(x_{n}-x^{*}\right)\right\rangle & =\lim _{j \rightarrow \infty}\left\langle(\gamma f-\mu F) x^{*}, j_{q}\left(x_{n_{j}}-x^{*}\right)\right\rangle \\
& =\left\langle(\gamma f-\mu F) x^{*}, j_{q}\left(z-x^{*}\right)\right\rangle \leq 0 .
\end{aligned}
$$

Step 5. Finally, we show that $\lim _{n \rightarrow \infty}\left\|x_{n}-x^{*}\right\|=0$. Setting $h_{n}=\beta_{n} \gamma f x_{n}+\left(I-\beta_{n} \mu F\right) S_{n} y_{n}$, $\forall n \geq 1$. Then we can rewrite $x_{n+1}=Q_{C} h_{n}$. It follows from Lemmas 2.1 and 2.4 that

$$
\begin{aligned}
& \left\|x_{n+1}-x^{*}\right\|^{q} \\
& \quad=\left\langle Q_{C} h_{n}-h_{n}, j_{q}\left(x_{n+1}-x^{*}\right)\right\rangle+\left\langle h_{n}-x^{*}, j_{q}\left(x_{n+1}-x^{*}\right)\right\rangle \\
& \quad \leq\left\langle h_{n}-x^{*}, j_{q}\left(x_{n+1}-x^{*}\right)\right\rangle
\end{aligned}
$$




$$
\begin{aligned}
= & \beta_{n}\left\langle\gamma f x_{n}-\mu F x^{*}, j_{q}\left(x_{n+1}-x^{*}\right)\right\rangle+\left\langle\left(I-\beta_{n} \mu F\right)\left(S_{n} y_{n}-x^{*}\right), j_{q}\left(x_{n+1}-x^{*}\right)\right\rangle \\
= & \beta_{n}\left\langle\gamma\left(f x_{n}-f x^{*}\right), j_{q}\left(x_{n+1}-x^{*}\right)\right\rangle+\beta_{n}\left\langle\gamma f x^{*}-\mu F x^{*}, j_{q}\left(x_{n+1}-x^{*}\right)\right\rangle \\
& +\left\langle\left(I-\beta_{n} \mu F\right)\left(S_{n} y_{n}-x^{*}\right), j_{q}\left(x_{n+1}-x^{*}\right)\right\rangle \\
\leq & \beta_{n} \gamma L\left\|x_{n}-x^{*}\right\|\left\|x_{n+1}-x^{*}\right\|^{q-1}+\beta_{n}\left\langle\gamma f x^{*}-\mu F x^{*}, j_{q}\left(x_{n+1}-x^{*}\right)\right\rangle \\
& +\left(1-\beta_{n} \tau\right)\left\|y_{n}-x^{*}\right\|\left\|x_{n+1}-x^{*}\right\|^{q-1} \\
\leq & \beta_{n} \gamma L\left\|x_{n}-x^{*}\right\|\left\|x_{n+1}-x^{*}\right\|^{q-1}+\beta_{n}\left\langle\gamma f x^{*}-\mu F x^{*}, j_{q}\left(x_{n+1}-x^{*}\right)\right\rangle \\
& +\left(1-\beta_{n} \tau\right)\left\|x_{n}-x^{*}\right\|\left\|x_{n+1}-x^{*}\right\|^{q-1} \\
= & \left(1-(\tau-\gamma L) \beta_{n}\right)\left\|x_{n}-x^{*}\right\|\left\|x_{n+1}-x^{*}\right\|^{q-1}+\beta_{n}\left\langle\gamma f x^{*}-\mu F x^{*}, j_{q}\left(x_{n+1}-x^{*}\right)\right\rangle \\
\leq & \left(1-(\tau-\gamma L) \beta_{n}\right)\left[\frac{1}{q}\left\|x_{n}-x^{*}\right\|^{q}+\frac{q-1}{q}\left\|x_{n+1}-x^{*}\right\|^{q-1}\right] \\
& +\beta_{n}\left\langle\gamma f x^{*}-\mu F x^{*}, j_{q}\left(x_{n+1}-x^{*}\right)\right\rangle,
\end{aligned}
$$

which implies that

$$
\begin{aligned}
\left\|x_{n+1}-x^{*}\right\|^{q} \leq & \frac{1-(\tau-\gamma L) \beta_{n}}{1+(q-1)(\tau-\gamma) \beta_{n}}\left\|x_{n}-x^{*}\right\|^{q} \\
& +\frac{q \beta_{n}}{1+(q-1)(\tau-\gamma L) \beta_{n}}+\left\langle\gamma f x^{*}-\mu F x^{*}, j_{q}\left(x_{n+1}-x^{*}\right)\right\rangle \\
\leq & \left(1-(\tau-\gamma L) \beta_{n}\right)\left\|x_{n}-x^{*}\right\|^{q} \\
& +\frac{q \beta_{n}}{1+(q-1)(\tau-\gamma L) \beta_{n}}+\left\langle\gamma f x^{*}-\mu F x^{*}, j_{q}\left(x_{n+1}-x^{*}\right)\right\rangle .
\end{aligned}
$$

Put $a_{n}=\beta_{n}(\tau-\gamma L)$ and $b_{n}=\frac{q}{\left(1+(q-1)(\tau-\gamma L) \beta_{n}\right)(\tau-\gamma L)}+\left\langle\gamma f x^{*}-\mu F x^{*}, j_{q}\left(x_{n+1}-x^{*}\right)\right\rangle$. Applying Lemma 2.3, we obtain $x_{n} \rightarrow x^{*}$ as $n \rightarrow \infty$. This completes the proof.

Remark 3.2 Theorem 3.1 improves and extends Theorem 2.1; see Cho and Kang [21]. Especially, our results extend the above results from Hilbert space to a more general $q$ uniformly smooth Banach space.

\section{Competing interests}

The authors declare that they have no competing interests.

\section{Authors' contributions}

All authors contributed equally to the writing of this paper. All authors read and approved the final manuscript.

\section{Acknowledgements}

The authors wish to thank the referees for their helpful comments, which notably improved the presentation of this manuscript.

Received: 11 September 2014 Accepted: 2 January 2015 Published online: 01 February 2015

\section{References}

1. Takahashi, W: Nonlinear Functional Analysis. Yokohama Publishers, Yokohama (2000)

2. Browder, FE, Petryshyn, WV: Construction of fixed points of nonlinear mapping in Hilbert space. J. Math. Anal. 20, 197-228 (1967)

3. Kinderlehrer, D, Stampacchia, G: An Introduction to Variational Inequalities and Their Applications. Academic Press, New York (1980) 
4. Noor, MA: General variational inequalities and nonexpansive mappings. J. Math. Anal. Appl. 331, 810-822 (2007)

5. Marino, G, Xu, HK: A general iterative method for nonexpansive mappings in Hilbert spaces. J. Math. Anal. Appl. 318 43-52 (2006)

6. Tian, M: A general iterative algorithm for nonexpansive mappings in Hilbert spaces. Nonlinear Anal., Theory Methods Appl. 73, 689-694 (2010)

7. Boonchari, D, Saejung, S: Weak and strong convergence theorems of an implicit iteration for a countable family of continuous pseudocontractive mappings. J. Comput. Appl. Math. 233, 1108-1116 (2009)

8. Boonchari, D, Saejung, S: Construction of common fixed points of a countable family of $\lambda$-demicontractive mappings in arbitrary Banach spaces. Appl. Math. Comput. 216, 173-178 (2010)

9. Yamada, I: The hybrid steepest descent method for the variational inequality problems over the intersection of fixed point sets of nonexpansive mappings. In: Butnariu, D, Censor, Y, Reich, S (eds.) Inherently Parallel Algorithms in Feasibility and Optimization and Their Applications. Stud. Comput. Math., vol. 8, pp. 473-504. North-Holland, Amsterdam (2001)

10. Takahashi, W, Toyoda, M: Weak convergence theorems for nonexpansive mappings and monotone mappings. J. Optim. Theory Appl. 118, 417-428 (2003)

11. Aoyama, K, Kimura, Y, Takahashi, W, Toyoda, M: Approximation of common fixed points of a countable family of nonexpansive mapping in a Banach space. Nonlinear Anal.. Theory Methods Appl. 67, 2350-2360 (2007)

12. Reich, S: Asymptotic behavior of contractions in Banach spaces. J. Math. Anal. Appl. 44, 57-70 (1973)

13. Song, Y, Luchuan, C: A general iteration scheme for variational inequality problem and common fixed point problems of nonexpansive mappings in q-uniformly smooth Banach spaces. J. Glob. Optim. 57, 1327-1348 (2013)

14. Bruck, RE: Properties of fixed point sets of nonexpansive mappings in Banach spaces. Trans. Am. Math. Soc. 179, 251-262 (1973)

15. Xu, HK: Iterative algorithm for nonlinear operators. J. Lond. Math. Soc. 2, 1-17 (2002)

16. Mitrinović, DS: Analytic Inequalities. Springer, New York (1970)

17. Xu, HK: Inequalities in Banach spaces with applications. Nonlinear Anal. 16, 1127-1138 (1991)

18. Kamimura, S, Takahashi, W: Strong convergence of a proximal-type algorithm in a Banach space. SIAM J. Optim. 13, 938-945 (2002)

19. Pongsakorn, S, Poom, K: Iterative methods for variational inequality problems and fixed point problems of a countable family of strict pseudo-contractions in a q-uniformly smooth Banach space. Fixed Point Theory Appl. (2012). doi:10.1186/1687-1812-2012-65

20. Chang, SS: On Chidumes open questions and approximate solutions of multivalued strongly accretive mapping equations in Banach spaces. J. Math. Anal. Appl. 216, 94-111 (1997)

21. Cho, SY, Kang, SM: Approximation of common solutions of variational inequalities via strict pseudo-contractions. Acta Math. Sci. 32(4), 1607-1618 (2012)

\section{Submit your manuscript to a SpringerOpen ${ }^{\odot}$ journal and benefit from:}

- Convenient online submission

- Rigorous peer review

Immediate publication on acceptance

- Open access: articles freely available online

- High visibility within the field

- Retaining the copyright to your article 